\title{
The significance of MAGED4 expression in non-small cell lung cancer as analyzed by real-time fluorescence quantitative PCR
}

\author{
QIN-YUN MA, LIE-WEN PANG, ZHI-MING CHEN, YONG-JUN ZHU, GANG CHEN and JI CHEN \\ Department of Thoracic Surgery, Huashan Hospital Affiliated to Fudan University, Shanghai 200040, P.R. China
}

Received March 14, 2012; Accepted June 25, 2012

DOI: $10.3892 / \mathrm{ol} .2012 .786$

\begin{abstract}
The aim of this study was to detect differences in the expression levels of melanoma-associated antigen D4 (MAGED4) mRNA between non-small cell lung cancer (NSCLC) tissues and normal tissues, and to compare differences in the expression levels of MAGED4 in tumor patients. Patients were grouped according to age, gender, smoking history, tumor size, pathological classification, degree of lung cancer cell differentiation and presence of lymph node metastasis. The expression levels of MAGED4 were detected using real-time fluorescence quantitative PCR. MAGED4 expression was higher in squamous cell carcinomas compared to adenocarcinomas $(\mathrm{P}<0.05)$, in poorly differentiated tissues compared to well-differentiated tissues $(\mathrm{P}<0.05)$, and in patients with lymph node metastasis compared to patients without lymph node metastasis $(\mathrm{P}<0.05)$. MAGED4 may be used as a specific antigen for NSCLC to influence the improvement of diagnosis, prognosis and immunological therapy outcomes in lung cancer patients.
\end{abstract}

\section{Introduction}

Lung cancer is one of the most common types of malignant tumor, with a morbidity rate that has increased during recent years (1). Non-small cell lung cancer (NSCLC) accounts for approximately $80-85 \%$ of all lung cancer cases (2-4). To date, the most effective therapeutic measure for treating this type of lung cancer has been surgical excision of the tumor; however, its efficacy is not always satisfactory, particularly in patients with advanced lung cancer for whom the five-year survival rate is relatively low (15-20\%) (3-5). Early diagnosis of lung cancer is not easy, and chances of overall survival greatly diminish as the cancer becomes more advanced. Therapeutic measures for detecting and treating lung cancer

Correspondence to: Dr Lie-Wen Pang, Department of Thoracic Surgery, Huashan Hospital Affiliated to Fudan University, 12 Mid Wulumuqi Road, Shanghai 200040, P.R. China

E-mail: chenzm20100831@hotmail.com

Key words: MAGED4 gene, non-small cell lung cancer, polymerase chain reaction have developed rapidly. Methods such as radiotherapy and chemotherapy are constantly improving and new chemotherapeutic drugs are continuously emerging. Overall, the therapeutic efficacy of existing methods for the treatment of lung cancer remains unsatisfactory. Therefore, it is necessary to explore new diagnostic and therapeutic methods to further improve the outcomes for lung cancer patients.

Currently, great attention has been paid to the expression of tumor-specific antigen genes and their association with tumorigenesis. The majority of these studies have evaluated melanoma-associated antigens (MAGEs). A member of this family, MAGED4, has been shown to be highly expressed in NSCLC. Therefore, we consider that the MAGED4 gene may be used as a specific antigen for NSCLC, and aid in the early diagnosis and successful treatment of patients with NSCLC.

\section{Patients and methods}

Patient data. Among the 54 patients with NSCLC, 33 (61.11\%) were male and $21(38.89 \%)$ were female. Patient ages ranged from $31-78$ years, with an average age of 57.33 years $( \pm 10.91)$. A total of $21(38.89 \%)$ patients had a smoking history of $\geq 20$ years (and $\geq 1$ pack/day), and $33(61.11 \%$ ) patients had a smoking history of $<20$ years (or $<1$ pack/day).

This study was approved by the Institutional Review Board of Zhongshan Hospital of Fudan University and all patients signed informed consent forms.

Tissue samples. All tissue samples were obtained by surgical excision from patients with pulmonary malignant tumors during their hospitalization at the Department of Thoracic Surgery of the Huashan Hospital (Shanghai, China). A total of 54 cases were pathologically diagnosed as primary malignant lung tumors (NSCLC), of which 34 (62.96\%) had a tumor size of $>3 \mathrm{~cm}$ and $20(37.04 \%)$ had a tumor size of $\leq 3 \mathrm{~cm}$. A total of $19(35.18 \%)$ cases were classified as squamous cell carcinoma, $31(57.41 \%)$ were classified as adenocarcinoma, three $(5.56 \%)$ were borderline undifferentiated large cell carcinoma and one $(1.85 \%)$ was pulmonary malignant melanoma. The tumor cells were well-differentiated in $17(31.48 \%)$ cases, moderately differentiated in $25(59.25 \%)$ cases and poorly differentiated in $12(9.00 \%)$ cases. Lymph node metastasis occurred in $26(48.15 \%)$ cases, the remaining $28(51.85 \%)$ cases had no lymph node metastasis. All patients underwent macroscopical radical resection and lymph node clearance. Once the tumors 
were excised, two sections of tissue of approximately $1 \mathrm{~cm}^{3}$ were removed; one from the tumor area and the other from an area $5 \mathrm{~cm}$ away from the tumor boundary, which was used as the non-cancerous tissue sample. The tissues were immediately preserved in liquid nitrogen at $-196^{\circ} \mathrm{C}$. Non-cancerous tissues were collected from 20 cases. None of the patients had received any antitumor treatments, including radiotherapy or chemotherapy, prior to the surgery.

The patients were grouped according to their age, gender, smoking history, tumor size, pathological classification, degree of lung cancer cell differentiation and presence of lymph node metastasis. The patient data for the tissue samples are shown in Table I.

Real-time fluorescence quantitative polymerase chain reaction (qPCR) was used to detect MAGED4 expression in the tumor and normal tissues according to the data collected $(6,7)$, and to analyze the correlation between the various groups.

Reagents and instruments. TRIzol reagent (Invitrogen Life Technologies, Carlsbad, CA, USA) was used for RNA extraction, and RNase-Free DNase M610A (Promega Corporation, Madison WI, USA) was used for DNA treatment. The Reverse Transcription System A3500 (Promega Corporation) was used for reverse transcription (RT) reactions, and SYBR-Green PCR Master mix (Cat No. 4309155; Applied Biosystems, Carlsbad, CA, USA) was used for qPCR. An ABI7900 fluorescence quantitative PCR instrument (Applied Biosystems) was used for PCR analysis.

Methods. RT-PCR was conducted to detect the expression level of MAGED4 mRNA. The primer sequences for MAGED4 were as follows: positive-sense strand, 5'-CCAGAATCAGAA CCGAGA-3' and antisense strand, 5'-CCAAAATCTCCG TCCTCA-3'; internal reference glyceraldehyde 3-phosphate dehydrogenase (GAPDH) positive-sense strand, 5'-TGAACG GGAAGCTCACTGG-3' and antisense strand, 5'-TCCACC ACCCTGTTGCTGTA-3'. Primers were synthesized by Shanghai Shenggong Biotechnology Co. (Shanghai, China). Total RNA extraction using an RNA extraction kit (Invitrogen Life Technologies) was conducted according to the manufacturer's instructions. The RT kit was from Promega Corporation, and total RNAs were reverse transcribed into cDNA according to the manufacturer's instructions. Real-time PCR was conducted using the Applied Biosystem high-throughput fluorescent quantitative PCR system $(7900 \mathrm{HT})$. The conditions for PCR reactions were as follows: $50^{\circ} \mathrm{C}$ for $2 \mathrm{~min}, 95^{\circ} \mathrm{C}$ for $10 \mathrm{~min}, 95^{\circ} \mathrm{C}$ for $15 \mathrm{sec}, 60^{\circ} \mathrm{C}$ for $1 \mathrm{~min}, 95^{\circ} \mathrm{C}$ for $15 \mathrm{sec}, 60^{\circ} \mathrm{C}$ for $15 \mathrm{sec}$ and $95^{\circ} \mathrm{C}$ for $15 \mathrm{sec}$. A total of 40 cycles were conducted and the values of MAGED4 and GAPDH were determined.

Correction of real-time PCR analysis. For real-time qPCR, all 74 sample volumes were $1 \mu \mathrm{l}$. Since the cDNA concentrations in each sample were not identical, possibly due to errors in concentration quantitation or the RT efficiency of RNA, results were normalized to GAPDH expression. $\triangle \mathrm{Ct}$ was obtained by subtracting the measured value of GAPDH from the measured value of MAGED4 and a $\Delta \mathrm{Ct}$ value was set as the standard value. Thus, $\Delta \Delta \mathrm{Ct}$ was obtained by comparing the $\Delta \mathrm{Ct}$ values of other samples, and $2^{-\Delta \Delta C \mathrm{t}}$ was obtained after
Table I. Data from 54 NSCLC patients.

\begin{tabular}{lcc}
\hline Patient data & No. of cases & $\%$ \\
\hline Age (years) & & \\
$<60$ & 31 & 57.41 \\
$\geq 60$ & 23 & 42.59 \\
Gender & & \\
Male & 33 & 61.11 \\
Female & 21 & 38.89 \\
Smoking history (years) & & \\
$\geq 20$ (and $\geq 1$ pack/day) & 21 & 38.89 \\
$<20$ (or <1 pack/day) & 33 & 61.11 \\
Tumor size (diameter, cm) & & \\
$\leq 3$ & 20 & 37.04 \\
$>3$ & 34 & 62.96 \\
Pathological classification & & \\
Squamous cell carcinoma & 19 & 35.18 \\
Adenocarcinoma & 31 & 57.41 \\
Other & 4 & 7.41 \\
Cell differentiation (group) & & \\
Well (a) & 17 & 31.48 \\
Moderate (b) & 25 & 59.52 \\
Poor (c) & 12 & 9.00 \\
Lymph node metastasis & & \\
$\mathrm{N}_{0}$ & 28 & 51.85 \\
$\mathrm{~N}_{1-2}$ & 26 & 48.15 \\
\hline
\end{tabular}

NSCLC, non-small cell lung cancer.

Table II. MAGED4 expression levels in NSCLC and normal lung tissues $(n=74)$.

\begin{tabular}{lccc}
\hline Tissue & $\begin{array}{c}\text { No. of } \\
\text { cases }\end{array}$ & $\begin{array}{c}\text { Mean MAGED4 } \\
\text { expression }\end{array}$ & P-value \\
\hline NSCLC & 54 & $1.74 \pm 1.07$ & $<0.01$ \\
Normal lung & 20 & $0.55 \pm 0.22$ & \\
\hline
\end{tabular}

MAGED4, melanoma-associated antigen D4; NSCLC, non-small cell lung cancer.

comparison and conversion using formulas. Finally, the relative expression level of MAGED4 mRNA in the sample was obtained (7).

Statistical analysis. All statistical analyses were conducted using SPSS software version 11.5 (SPSS Inc., Chicago, IL, USA). Analysis of variance (ANOVA) and the Student-Newman-Keuls test (q-test) were used for statistical analysis of data between multiple groups, and a t-test was used to evaluate the difference in means between the two groups. $\mathrm{P}<0.05$ was used to indicate a statistically significant difference. 


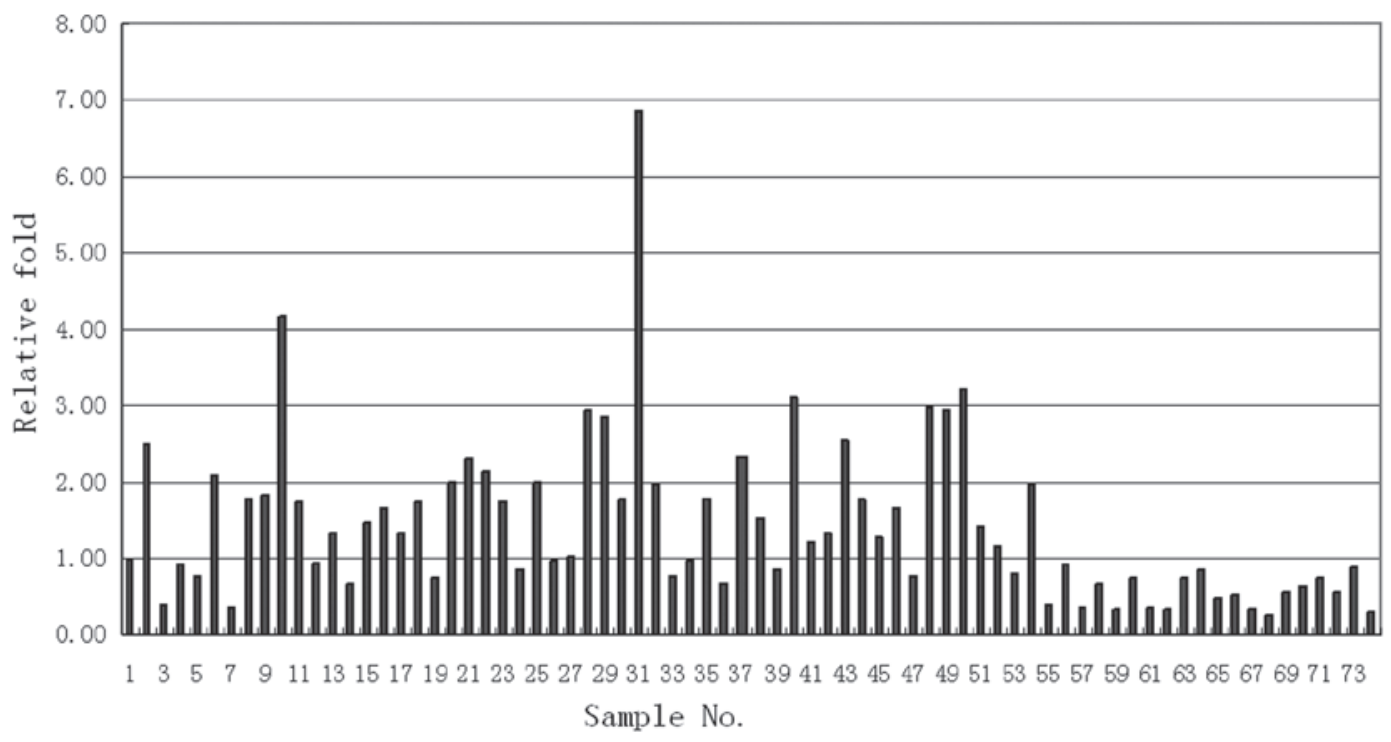

Figure 1. Column diagram revealing the changes in the measured values of MAGED4 mRNA. Expression levels are significantly higher in the 54 NSCLC tissues compared to those of the 20 normal lung tissues. GAPDH was used as a reference control. qPCR, quantitative polymerase chain reaction; GAPDH, glyceraldehyde 3-phosphate dehydrogenase.

\section{Results}

The measured MAGE4 values of the 74 patients are shown in Fig. 1. The X-axis represents the number of cases and the Y-axis represents the corresponding measured value of MAGED4 for each patient. We identified that the measured values of MAGED4 in the 54 NSCLC tissues were significantly higher compared to those of the 20 normal lung tissues (Fig. 1).

Statistical analysis revealed that the expression level of MAGED4 in tumor tissues (mean, 1.74 \pm 1.07 ) was significantly higher than that of normal lung tissues, non-cancerous tissues excised more than $5 \mathrm{~cm}$ away from the focus, (mean, $0.55 \pm 0.22 ; \mathrm{P}<0.01$; Table II).

MAGED4 expression in NSCLC tumor tissues was then compared between the various groups. The results revealed that MAGED4 expression was not significantly correlated to patient age, gender, smoking history or tumor size ( $\mathrm{P}>0.05)$. Notably, MAGED4 expression in squamous cell carcinoma was significantly higher than that in adenocarcinoma $(\mathrm{P}<0.01)$. In the various cell differentiation types, expression levels of MAGED4 were relatively higher when the cells were poorly differentiated compared to when the cells were well differentiated. The expression levels of MAGED4 increased from poorly differentiated cells to well-differentiated cells, with moderately differentiated cells having an intermediate level of expression $(\mathrm{P}<0.01)$. MAGED4 expression levels in patients with lymph node metastasis were significantly higher compared to patients without lymph node metastasis $(\mathrm{P}<0.05$; Table III).

\section{Discussion}

Lung cancer is one of the leading causes of mortality. With continually rising rates of morbidity and mortality, lung cancer has become the leading cause of mortality among carcinomas in numerous countries worldwide (1). Although a vast amount of research has been conducted regarding early prevention, diagnosis and clinical treatments of lung cancer, the five-year survival rate remains unsatisfactory (approximately $15 \%$ ) (2-4). Therefore, there is an urgent requirement to identify new diagnostic and therapeutic methods for the treatment of lung cancer.

Genetic diagnosis and treatments for lung cancer may be one direction for the development of diagnosis and treatments for lung cancer in the future. MAGEs are a group of tumorassociated antigens (TAA), which were first discovered in melanomas (8). The MAGE gene family is almost completely silent in normal tissues and is mainly expressed in malignant tumor tissues, particularly in melanomas. MAGE expression correlates with tumor specificity to a relatively high degree (9), and its expression is closely associated with the development, progression and prognosis of tumors. Thus, its products may be used in molecular diagnosis and immunotherapy of tumors. Van der Bruggen et al discovered the first MAGE, MAGE1, during a study of malignant melanomas in 1991 (8). Following this study, attempts were made to further characterize other members of the MAGE gene family.

Previous studies revealed that MAGE antigens were expressed in various malignant tumors (10-15), while there was low or no expression of MAGE in normal tissues. Certain MAGE family members also demonstrated relatively high levels of positive expression in lung cancer tissues. Certain studies indicate that the positive expression rate of MAGE-A3 in lung cancer is as high as $30-50 \%(16,17)$. However, findings obtained by other studies vary widely. One study revealed that MAGE-A3 expression levels in lung cancer were only $13 \%$ (18). Identification of a consistent lung cancer-specific antigen remains an aim for researchers in the field.

MAGED4 is a member of the MAGE gene family that has been recently discovered (19). In contrast to other MAGE 
Table III. MAGED4 gene expression in NSCLC tumor tissues compared between various patient groups $(\mathrm{n}=54)$.

\begin{tabular}{|c|c|c|c|}
\hline Patient data & $\begin{array}{l}\text { No. of cases } \\
(\%)\end{array}$ & $\begin{array}{c}\text { Mean MAGED4 } \\
\text { expression }\end{array}$ & P-value \\
\hline \multicolumn{4}{|l|}{ Age (years) } \\
\hline$<60$ & $31(57.41)$ & $1.96 \pm 1.29$ & \multirow[t]{2}{*}{0.08} \\
\hline$\geq 60$ & $23(42.59)$ & $1.44 \pm 0.60$ & \\
\hline \multicolumn{4}{|l|}{ Gender } \\
\hline Male & $33(61.11)$ & $1.75 \pm 0.85$ & \multirow[t]{2}{*}{0.92} \\
\hline Female & $21(38.89)$ & $1.72 \pm 1.39$ & \\
\hline \multicolumn{4}{|l|}{ Smoking history (years) } \\
\hline$\geq 20$ (and $\geq 1$ pack/day) & $21(38.89)$ & $1.73 \pm 0.82$ & \multirow[t]{2}{*}{0.95} \\
\hline <20 (or <1 pack/day) & $33(61.11)$ & $1.75 \pm 1.22$ & \\
\hline \multicolumn{4}{|l|}{ Tumor size (cm) } \\
\hline$\leq 3$ & $20(37.04)$ & $1.52 \pm 0.63$ & \multirow[t]{2}{*}{0.27} \\
\hline$>3$ & $34(62.96)$ & $1.87 \pm 1.26$ & \\
\hline \multicolumn{4}{|l|}{ Pathological classification } \\
\hline Squamous cell carcinoma & $19(34.55)$ & $1.99 \pm 0.74$ & \multirow{3}{*}{$\begin{array}{l}\text { Not included in } \\
\text { statistical analysis }\end{array}$} \\
\hline Adenocarcinoma & $32(58.18)$ & $1.27 \pm 0.53$ & \\
\hline Other & $4(7.27)$ & $4.23 \pm 1.85$ & \\
\hline \multicolumn{4}{|l|}{ Cell differentiation (group) } \\
\hline Well (a) & $17(31.48)$ & $1.01 \pm 0.45$ & \multirow{3}{*}{$\begin{array}{c}<0.01 \\
(\mathrm{c}>\mathrm{b}>\mathrm{a})^{\mathrm{a}}\end{array}$} \\
\hline Moderate (b) & $25(46.29)$ & $1.65 \pm 0.59$ & \\
\hline Poor $(c)$ & $12(22.22)$ & $2.96 \pm 1.44$ & \\
\hline \multicolumn{4}{|l|}{ Lymph node metastasis } \\
\hline $\mathrm{N}_{0}$ & $28(51.85)$ & $1.42 \pm 0.60$ & \multirow[t]{2}{*}{0.031} \\
\hline $\mathrm{N}_{1-2}$ & $26(48.15)$ & $2.07 \pm 1.36$ & \\
\hline
\end{tabular}

MAGED4, melanoma-associated antigen D4; NSCLC, non-small cell lung cancer. ${ }^{\text {a} E x p r e s s i o n ~ l e v e l s ~ o f ~ M A G E D 4 ~ w e r e ~ s i g n i f i c a n t l y ~ h i g h e s t ~}$ in group c, followed by group b, then group a.

members, MAGED4 demonstrates low levels of expression in many malignant tumors outside of the lung, while its expression level in NSCLC is relatively high (19).

Our study compared the expression levels of MAGED4 between 54 cases of surgically-resected NSCLC and non-cancerous lung tissues proximal to the cancer using real-time fluorescence qPCR. Expression of MAGED4 in normal tissues was significantly lower compared to that in tumor tissues ( 0.55 vs. $1.74 ; \mathrm{P}<0.01)$.

Previous studies have reported that genes in the MAGED4 family may be detected at a significantly increased level in the majority of malignant tumor tissues. This is due to the characterization of malignant tumors by melanoma, and due to the fact that low levels that are typically found in normal tissues, with the exception of the testicles and placenta (20). However, Jang et al discovered that MAGE mRNA was detected in corresponding normal tissues (21), and in 2006 Ito et al also revealed that MAGED4 expression was detected at low levels in normal tissues (19). These results correspond with our findings in the present study which indicate that MAGED4 expression can be detected at low levels in normal tissues. We suggest that the reason for this discrepancy may be due to the slightly different expression features of various members of the MAGE family in tissues. It may also be related to the processing of the tissue samples, methods for experimental detection or other factors. The real-time fluorescence qPCR detection in this study had a higher sensitivity and accuracy in comparison to previous staining, immunohistological methods and traditional PCR detection. Its high sensitivity is able to detect less than 10 tumor cells from $1 \times 10^{5}-1 \times 10^{7}$ nucleated cells; thus, it is a well-received accurate RNA detection technique.

We conducted a more detailed analysis for the high expression levels of MAGED4 in NSCLC and revealed that expression in NSCLC tissues was not significantly correlated with patient age or gender $(\mathrm{P}>0.05)$. These results were in accordance with the properties of other members of the MAGE gene family discovered in previous studies $(15,17,18)$.

Since the development of lung cancer has previously been shown to be correlated with smoking (22), we divided patients into two groups; those with a smoking history of $\geq 20$ years and those with a smoking history of $<20$ years. Notably, the expression levels of MAGED4 in these two groups did not demonstrate a significant difference $(\mathrm{P}>0.05)$. Ito et al similarly revealed that MAGED4 expression level did not correlate with patient smoking history (19); while a number of earlier 
studies on other members of the MAGE gene family, including MAGE-A1, -A3 and -B2, indicated that the activation and expression of these genes had a certain degree of correlation with smoking (21). We suggest that the discrepancy in these findings is due to different biological behaviors of the various members of the MAGE gene family. We presume that the reason for this result may be attributed to the difference in biological behaviors in various genes in the MAGE family.

This study demonstrated that expression of MAGED4 in the younger age group was higher than that of the older age group (1.96 vs. 1.44; $\mathrm{P}>0.05$ ). This difference in expression may be attributed to the slightly more rapid tumor cell proliferation in young patients due to an increased cellular activity (19). Studies have revealed that the proliferation speed of tumor cells has a certain degree of correlation with expression of the MAGE gene family (19). However, we identified that there was no significant difference between the two age groups $(\mathrm{P}>0.05)$, which may be due to the relatively small sample size used in this study.

Members of the MAGE gene family have been identified to have higher expression levels in tumors with relatively larger diameters (23). In this study, we discovered that MAGED4 expression was not significantly correlated with tumor size $(\mathrm{P}>0.05)$. This may result from the different expression characteristics of the various subtypes of genes in the MAGE family. Research data for MAGED4 regarding this topic requires further investigation.

Currently, there is no agreement with regards to the correlation between MAGE expression and pathological types of tumors. Yoshimatsu et al revealed that the expression levels of MAGE-1 and -3 were higher in squamous cell carcinomas compared to adenocarcinomas (15), while Shen et al revealed that there was no significant difference in MAGE-1 expression between these two types of carcinomas (24). There have been few studies focusing on the correlation between MAGED4 expression and pathological types of tumors. In accordance with the results of this study, Ito et al identified that expression levels of MAGED4 in squamous cell carcinomas were higher than those in adenocarcinomas (19). The difference in expression levels may be due to the different proliferation profiles and molecular genetic characteristics between squamous cell carcinoma and adenocarcinoma cells.

In the present study, there was a certain degree of correlation between the expression level of MAGED4 and the differentiation degree of tumor cells. The expression level of MAGED4 was higher when the tumor cells were poorly differentiated and lower when the tumor cells were well differentiated. We categorized the pathological sections of the samples into three groups according to the tumor cell differentiation degree: a, well-differentiated carcinoma; b, moderately differentiated carcinoma; c, poorly differentiated carcinoma. Multiple comparisons demonstrated that expression levels of MAGED4 were highest in $\mathrm{c}$, followed by $\mathrm{b}$, then a $(\mathrm{P}<0.01)$. This result provides a general guideline that may be helpful in selecting therapies and providing prognostic information for NSCLC patients.

It is well-known that the metastatic status of lymph nodes is an important prognostic factor in cancer; therefore, we categorized the samples into two groups according to whether lymph node metastasis had occurred. Our results demonstrated that the expression level of MAGED4 in the lymph node metastasis group was higher than that in the group without lymph node metastasis $(\mathrm{P}<0.05)$. In the literature, there has been no agreement in terms of the correlation between MAGE gene family expression and metastatic status of lymph nodes. Dango et al reported that the expression of MAGE-A was correlated with lymph node metastasis (25), while Chang et al proposed that they were not correlated (26). The results of this study indicate that there is a certain degree of correlation between MAGED4 expression and the metastatic status of lymph nodes. These results will aid in providing prognostic information with regards to NSCLC.

In the present study, we discovered that MAGED4 expression in NSCLC tissues was significantly higher than that in normal lung tissues. This is in accordance with results from other members of the MAGE family. MAGE-A1, -A2 and -A3 demonstrated higher expression levels in malignant tumor tissues compared to normal tissues; however, the expression of MAGED4 in NSCLC tissues revealed its particularity as it did not follow the consistent expression of the other genes in the MAGE family.

The mechanism of MAGED4 activity in tumor cells also remains unclear. Studies have been conducted on more well-characterized family members, including MAGE-A1, -A2 and -A3, but further study is required in order to compare MAGED4 to these family members. Studies on these genes have entered the stage of immunotherapy investigation. Studies on active specific immunotherapy with MAGE peptide-based tumor vaccines and adoptive therapy with in vitro-induced MAGE-specific cytotoxic T lymphocyte (CTL) of certain MAGE-positive cancers have been conducted, and a degree of efficacy has been demonstrated $(27,28)$.

In conclusion, MAGED4 is a newly discovered member of the MAGE gene family. Its expression level in NSCLC is higher than that of other members of the MAGE family, but studies on the MAGED4 gene remain insufficient (29). In our study, the expression of MAGED4 in NSCLC tissues revealed a certain degree of specificity and sensitivity. The results may be beneficial for the diagnosis and treatments of NSCLC in the future.

\section{Acknowledgements}

This study was supported by the Youth Foundation of Fudan University and the Startup Foundation of Huashan Hospital, Fudan University.

\section{References}

1. Howe HL, Wingo PA, Thun MJ, et al: Annual report to the nation on the status of cancer (1973 through 1998), featuring cancers with recent increasing trends. J Natl Cancer Inst 93: 824-842, 2001.

2. Crinò L, Weder W, van Meerbeeck J, Felip E; ESMO Guidelines Working Group: Early stage and locally advanced (non-metastatic) non-small-cell lung cancer: ESMO clinical practice guidelines for diagnosis, treatment and follow-up. Ann Oncol 21: v103-v115, 2010

3. EttingerDS, Akerley W,Bepler G, Blum MG,Chang A, Cheney RT, Chirieac LR, D'Amico TA, Demmy TL, Ganti AK, et al; NCCN Non-Small Cell Lung Cancer Panel Members: Non-small cell lung cancer. J Natl Compr Canc Netw 8: 740-801, 2010.

4. Jemal A, Siegel R, Ward E, et al: Cancer statistics, 2008. CA Cancer J Clin 58: 71-96, 2008. 
5. Pisters KM, Vallières E, Crowley JJ, et al: Surgery with or without preoperative paclitaxel and carboplatin in early-stage non-small-cell lung cancer: Southwest Oncology Group Trial S9900, an intergroup, randomized, phase III trial. J Clin Oncol 28: 1843-1849, 2010.

6. Johnson VJ, Yucesoy B and Luster MI: Genotyping of single nucleotide polymorphisms in cytokine genes using real-time PCR allelic discrimination technology. Cytokine 27: 135-141, 2004.

7. Livak KJ and Schmittgen TD: Analysis of relative gene expression data using real-time quantitative PCR and the 2(-Delta Delta C(T)) method. Methods 25: 402-408, 2001.

8. van der Bruggen $\mathrm{P}$, Traversari C, Chomez $\mathrm{P}$, et al: A gene encoding an antigen recognized by cytolytic $\mathrm{T}$ lymphocytes on a human melanoma. Science 254: 1643-1647, 1991.

9. De Plaen E, Arden K, Traversari C, et al: Structure, chromosomal localization, and expression of 12 genes of the MAGE family. Immunogenetics 40: 360-369, 1994

10. Kariyama K, Higashi T, Kobayashi Y, et al: Expression of MAGE- 1 and -3 genes and gene products in human hepatocellular carcinoma. Br J Cancer 81: 1080-1087, 1999.

11. Noguchi S, Aihara T, Motomura $\mathrm{K}$, Inaji $\mathrm{H}$, Imaoka $\mathrm{S}$ and Koyama H: Detection of breast cancer micrometastases in axillary lymph nodes by means of reverse transcriptase-polymerase chain reaction. Comparison between MUC1 mRNA and keratin 19 mRNA amplification. Am J Pathol 148: 649-656, 1996.

12. Ofuji S, Ikeda M, Tsujitani S, et al: Expression of MAGE-1, MAGE-2 and MAGE-3 genes in human gastric carcinomas; lack of evidence for cytotoxic effects in cases with simultaneous expression of MAGE-3 and HLA-A2. Anticancer Res 18 3639-3644, 1998.

13. Quillien V, Raoul JL, Heresbach D, Collet B, Toujas L and Brasseur F: Expression of MAGE genes in esophageal squamous-cell carcinoma. Anticancer Res 17: 387-391, 1997.

14. Sahin U, Koslowski M, Tureci O, et al: Expression of cancer testis genes in human brain tumors. Clin Cancer Res 6: 3916-3922, 2000.

15. Yoshimatsu T, Yoshino I, Ohgami A, et al: Expression of the melanoma antigen-encoding gene in human lung cancer. J Surg Oncol 67: 126-129, 1998

16. Grunwald C, Koslowski M, Arsiray T, et al: Expression of multiple epigenetically regulated cancer/germline genes in nonsmall cell lung cancer. Int J Cancer 118: 2522-2528, 2006.
17. Tajima K, Obata Y, Tamaki H, et al: Expression of cancer/testis (CT) antigens in lung cancer. Lung Cancer 42: 23-33, 2003.

18. Tsai JR, Chong IW, Chen YH, et al: Differential expression profile of MAGE family in non-small-cell lung cancer. Lung Cancer 56: 185-192, 2007.

19. Ito S, Kawano Y, Katakura H, et al: Expression of MAGE-D4, a novel MAGE family antigen, is correlated with tumor-cell proliferation of non-small cell lung cancer. Lung Cancer 51: 79-88, 2006.

20. Muscatelli F, Walker AP, De Plaen E, Stafford AN and Monaco AP: Isolation and characterization of a MAGE gene family in the Xp21.3 region. Proc Natl Acad Sci USA 92: 4987-4991, 1995

21. Jang SJ, Soria JC, Wang L, et al: Activation of melanoma antigen tumor antigens occurs early in lung carcinogenesis. Cancer Res 61: 7959-7963, 2001.

22. Parsons A, Daley A, Begh R and Aveyard P: Influence of smoking cessation after diagnosis of early stage lung cancer on prognosis: systematic review of observational studies with meta-analysis. BMJ 340: b5569, 2010.

23. Shichijo S, Hayashi A, Takamori S, et al: Detection of MAGE-4 protein in lung cancers. Int J Cancer 64: 158-165, 1995.

24. Shen C, Wang D, Jiang G, Liu J and Zhang G: mRNA expressions of MAGE genes in human lung cancer. Zhongguo Fei Ai Za Zhi 6: 268-271, 2003 (In Chinese).

25. Dango S, Cucuruz B, Mayer O, et al: Detection of disseminated tumour cells in mediastinoscopic lymph node biopsies and endobronchial ultrasonography-guided transbronchial needle aspiration in patients with suspected lung cancer. Lung Cancer 68: 383-388, 2010.

26. Chang HK, Park J, Kim W, et al: The expression of MAGE and GAGE genes in uterine cervical carcinoma of Korea by RT-PCR with common primers. Gynecol Oncol 97: 342-347, 2005.

27. Eifuku R, Takenoyama M, Yoshino I, et al: Analysis of MAGE-3 derived synthetic peptide as a human lung cancer antigen recognized by cytotoxic $\mathrm{T}$ lymphocytes. Int $\mathrm{J}$ Clin Oncol 6: 34-39, 2001.

28. Marchand M, van Baren N, Weynants P, et al: Tumor regressions observed in patients with metastatic melanoma treated with an antigenic peptide encoded by gene MAGE- 3 and presented by HLA-A1. Int J Cancer 80: 219-230, 1999.

29. Sang M, Wang L, Ding C, et al: Melanoma-associated antigen genes - an update. Cancer Lett 302: 85-90, 2011. 